\title{
Lung cancer cells release high mobility group box 1 and promote the formation of neutrophil extracellular traps
}

\author{
JIAWEI ZHOU ${ }^{1 *}$, YONGLIN YANG $^{2 *}$, TINGTING GAN $^{1}$, YAN LI $^{1}$, FAN HU $^{3}$, \\ NANNAN HAO ${ }^{4}$, BAORUI YUAN $^{4}, \mathrm{YU} \mathrm{CHEN}^{1}$ and MINGSHUN ZHANG ${ }^{4}$ \\ ${ }^{1}$ Department of Anesthesiology, First Affiliated Hospital of Nanjing Medical University, Nanjing, Jiangsu 210029; \\ ${ }^{2}$ Department of Infectious Diseases, Nanjing First Hospital, Nanjing Medical University, Nanjing, Jiangsu 210006; \\ ${ }^{3}$ Analysis Center and ${ }^{4}$ Department of Immunology, Nanjing Medical University, Nanjing, Jiangsu 210016, P.R. China
}

Received March 27, 2018; Accepted March 22, 2019

DOI: $10.3892 / \mathrm{ol} .2019 .10290$

\begin{abstract}
Lung cancer is the leading cause of cancer-associated mortality. Tumor-associated neutrophils represent a large portion of inflammatory cells within the lung tumor microenvironment. However, the roles of neutrophil extracellular traps (NETs) in lung cancer remain unclear. In the present study, it was identified that Lewis lung carcinoma cells actively released the danger-associated molecular pattern protein high mobility group box 1 (HMGB1). Furthermore, HMGB1 in lung cancer cell supernatants promoted the formation of neutrophil extracellular traps (NETs), which was dependent on Toll-like receptor 4 (TLR4). The downstream molecules of TLR4, including myeloid differentiation factor 88 , TIR-domain-containing adapter-inducing interferon- $\beta$, p38 mitogen-activated protein kinases (p38 MAPKs) and extracellular signal-regulated kinases (ERKs), were activated during the formation of NETs. In addition, inhibition of p38 MAPKs or ERKs significantly decreased NETs. Morphine, an additional ligand for TLR4, aggravated the NETs induced by lung cancer cells. The present study revealed novel mechanisms in tumor-associated NET formation.
\end{abstract}

\section{Introduction}

Lung cancer is one of the most devastating diseases worldwide. Neutrophil infiltration is frequently observed in lung cancer

Correspondence to: Dr Yu Chen, Department of Anesthesiology, First Affiliated Hospital of Nanjing Medical University, 300 Guangzhou Road, Nanjing, Jiangsu 210029, P.R. China

E-mail: yuchen_njmu@163.com

Dr Mingshun Zhang, Department of Immunology, Nanjing Medical University, 101 Longmian Road, Nanjing, Jiangsu 210016, P.R. China E-mail: mingshunzhang@njmu.edu.cn

${ }^{*}$ Contributed equally

Key words: lung cancer, neutrophil extracellular traps, high mobility group box 1, morphine, Toll-like receptor 4 tissues (1). Neutrophil extracellular traps (NETs), composed of extracellular DNA, hypercitrullined histones and antimicrobial enzymes from neutrophils, may increase the adhesion of cancer cells (2) and sequester lung cancer cells in the blood (3). NET formation has previously been described in patients with lung cancer (4). However, the mechanisms regulating the formation of NETs associated with lung cancer are yet to be fully elucidated.

Diverse stimuli have been suggested to initiate the formation of NETs, ranging from pathogen components (5) to neutrophil antibodies (6) and activated platelets (7). In addition, interleukin (IL)-1 $\beta$ (8), IL-8 (9) or granulocyte colony stimulating factor (G-CSF) (10) in the tumor microenvironment may also promote NET formation. As a damage-associated molecular pattern protein, high mobility group box 1 (HMGB1) serves a paradoxical role in regulating cell death and survival in tumor development (11). HMGB1 interactions with Toll-like receptor 4 (TLR4) have been demonstrated to induce NETs (12). Therefore, it was hypothesized that lung cancer cells may release HMGB1, which may induce NET formation.

Morphine is an effective analgesic for cancer-associated pain. In the end-stages of lung cancer, continuous morphine infusion is used to alleviate pain (13). Although pain adversely affects the prognosis of patients with lung cancer, morphine administration controls the pain but does not improve survival (14). Arguably, morphine may stimulate angiogenesis and promote tumor progression $(15,16)$. HGMB1 and morphine are able to bind with TLR4 $(17,18)$. The present study aimed to evaluate the role of HMGB1 from lung cancer cells in the formation of NETs. In addition, the effect of morphine on HMGB1-induced NETs was investigated.

\section{Materials and methods}

Animals and ethics statement. In total, 40 wild-type female ICR mice (age matched 8-10 weeks old) weighing 29-32 g were purchased from Yangzhou University (Yangzhou, China) and bred in the animal facility of Nanjing Medical University (Nanjing, China) under standard laboratory conditions (12:12 h light:dark cycle, relative humidity $60 \pm 5 \%$, temperature $25 \pm 2^{\circ} \mathrm{C}$ ) in individually ventilated cages without restriction to water or food. All animal procedures were approved by The 
Institutional Animal Care Committee of Nanjing Medical University.

Lewis lung carcinoma (LLC) cell culture and flow cytometry. The murine LLC cell line was purchased from the Cell Bank of the Shanghai Institute for Biological Sciences, Chinese Academy of Sciences. LLC cells were maintained in high-glucose Dulbecco's modified Eagle's medium (HyClone; GE Healthcare Life Sciences) supplemented with penicillin (100 U/ml), streptomycin (100 $\mu \mathrm{g} / \mathrm{ml})$ and 10\% FBS (Gibco; Thermo Fisher Scientific, Inc.) in a $5 \% \mathrm{CO}_{2}$ humidified atmosphere at $37^{\circ} \mathrm{C}$.

Cells which had adhered to the base of the T-25 flask were dislodged by aspiration several times with culture medium. The supernatants were used to stimulate neutrophils for NETs as described subsequently. The LLC cells were resuspended in PBS buffer with 1\% BSA (Sigma-Aldrich; Merck KGaA). Aliquots containing $1 \times 10^{5}$ cells in $100 \mu \mathrm{l}$ buffer were stained with $10-\mu 1$ propidium iodide $(50 \mathrm{mg} / \mathrm{ml})$ solution and with $5 \mu \mathrm{l}$ fluorescein isothiocyanate-conjugated Annexin V $(17.6 \mathrm{mg} / \mathrm{ml}$; $\mathrm{BD}$ Biosciences) for $5 \mathrm{~min}$ at $37^{\circ} \mathrm{C}$. Following staining, $400 \mu \mathrm{l}$ PBS was added to the cells. Immediately, flow cytometry analysis was performed using a FACScan flow cytometer (BD Biosciences). All FACS data were analyzed using FlowJo v10 software (FlowJo LLC).

Quantification of NETs released from neutrophils. Terminal anesthesia was performed by intraperitoneal injection of a mixture of $10 \mathrm{mg} / \mathrm{kg}$ xylazine (MTC Pharmaceuticals) and $100 \mathrm{mg} / \mathrm{kg}$ ketamine hydrochloride (Rogar/STB; Pfizer Canada, Inc.) prior to sacrifice by cervical dislocation. Following sacrifice, the femur and the tibia from the two hind legs were removed and the extreme distal tip of each extremity was cut off. PBS solution was forced through the bone with a $1 \mathrm{ml}$ syringe. Following ammonium chloride erythrocyte lysis, murine neutrophils were prepared by Histopaque-based density gradient centrifugation, as described previously (19).

Murine neutrophils were unstimulated or challenged with LLC cell supernatants for 2, 4 or $8 \mathrm{~h}$. Following incubation, the non-cell-permeable DNA dye Sytox Green (5 $\mu \mathrm{M}$; Invitrogen; Thermo Fisher Scientific, Inc.) was used to quantify the released NETs in the supernatants as described previously (20). The samples were examined with a fluorometric reader Infiniti M200 (Tecan Group, Ltd.) using an excitation wavelength of $488 \mathrm{~nm}$ and an emission wavelength of $523 \mathrm{~nm}$, as described previously (4).

Concomitantly, prior to stimulation with LLC cell supernatants, neutrophils were pretreated with the following specific inhibitors for $30 \mathrm{~min}$ at $37^{\circ} \mathrm{C}$ : HMGB1 inhibitor Glycyrrhizin glycyrrhizic acid (GA; $10 \mu \mathrm{M}$; Selleck Chemicals), TLR4 inhibitor C34 (10 $\mu \mathrm{M}$; Tocris Bioscience), extracellular signal-regulated kinase (ERK) inhibitor UO126 (50 $\mu \mathrm{M}$; Cell Signaling Technology, Inc.) and p38 mitogen-activated protein kinases (p38 MAPKs) inhibitor SB203580 (10 $\mu \mathrm{M}$; Selleck Chemicals). For the morphine experiments, neutrophils were pretreated with morphine (10 nM; Sigma-Aldrich; Merck $\mathrm{KGaA}$ ) or naloxone (100 nM; Sigma-Aldrich; Merck KGaA) for $30 \mathrm{~min}$ at $37^{\circ} \mathrm{C}$. Then, neutrophils were incubated with LLC cell supernatants for $4 \mathrm{~h}$.
Western blot analysis. Following incubation, neutrophils were collected and centrifuged for $5 \mathrm{~min}$ at $500 \mathrm{x} \mathrm{g}$ and $4^{\circ} \mathrm{C}$. Cell pellets were lysed in radioimmunoprecipitation assay lysis buffer (Beyotime Institute of Biotechnology) on ice for $5 \mathrm{~min}$. Then, the cell suspensions were centrifuged for $10 \mathrm{~min}$ at $12,000 \mathrm{x} \mathrm{g}$ and $4^{\circ} \mathrm{C}$. The supernatant was solubilized in 5X SDS-PAGE sample buffer and boiled for $10 \mathrm{~min}$. The protein concentration in each sample was quantified using a NanoDrop 2000 with NanoDrop One (v1.6; both from Thermo Fisher Scientific, Inc.) and $30 \mu \mathrm{g}$ protein per lane was resolved using a 10\% SDS-PAGE, transferred by electrophoresis onto a polyvinylidene fluoride membrane (EMD Millipore), blocked with 5\% BSA for $1 \mathrm{~h}$ at room temperature and probed with the following antibodies at 1:1,000 dilution overnight at $4^{\circ} \mathrm{C}$ : Anti-Histone $\mathrm{H} 3$ (cat. no. 4499s; Cell Signaling Technology, Inc.), anti-histone3 (cat. no. ab5103; citrulline R2+R8+R17; Abcam), MAPK/Phospho-MAPK family antibody (cat. no. 9926; Cell Signaling Technology, Inc.), anti-TIR-domain-containing adapter-inducing interferon- $\beta$ (TRIF) antibody (cat. no. ab13810, Abcam), anti-HMGB1 antibody (cat. no. 10829-1-AP, ProteinTech Group, Inc., Chicago, IL, USA) and anti-myeloid differentiation factor 88 (MyD88) antibody (cat. no. 4283; Cell Signaling Technology, Inc.). This was followed by incubation with horseradish peroxidase (HRP)-conjugated goat anti-rabbit IgG (dilution 1:10,000; cat. no. ab6721; Abcam) in PBS with $0.5 \%$ BSA for $1 \mathrm{~h}$ at room temperature. Signals were developed and analyzed using the chemiluminescent horseradish peroxidase substrate (EMD Millipore) and the G:BOX system (Syngene Europe). Grayscale analysis was performed using Adobe Photoshop CS6 (v13.0 x32; Adobe Systems Europe, Ltd., Maidenhead, UK).

Immunofluorescence microscopy. Neutrophils $\left(1 \times 10^{6}\right)$ were seeded on glass-bottomed dishes (Shanghai Jing An biological science and Technology Co., Ltd.). According to the aforementioned method, LLC cell supernatants or different inhibitors were added. Following $4 \mathrm{~h}$ of incubation, cells that adhered to the bottom of the glass were carefully fixed with ice-cold acetone $(\geq 99 \%)$ for $10 \mathrm{~min}$ at room temperature. The samples were blocked with $5 \%$ goat serum (cat. no. 16210072, Gibco, Thermo Fisher Scientific, Inc.) and stained overnight at $4{ }^{\circ} \mathrm{C}$ with rabbit polyclonal antibody against Histone3 (cat. no. ab5103, citrulline R2+R8+R17; 1:300; Abcam). The samples were washed in PBST and stained with Alexa Fluor ${ }^{\circledR} 555$ goat anti-rabbit antibody (1:500; cat. no. A-21428; Thermo Fisher Scientific, Inc.). DNA in the samples was stained with Sytox Green (Invitrogen; Thermo Fisher Scientific, Inc.; 1:10,000) for $30 \mathrm{~min}$ at room temperature. Images were captured using Carl Zeiss confocal microscopes (Carl Zeiss AG) with appropriate lenses and filters (magnification, 200x).

Statistical analysis. Statistical analysis was performed using GraphPad Prism 5 (GraphPad Software, Inc.). Data are presented as the mean \pm standard error of the mean. One-way analysis of variance and a post-hoc Tukey's honest significant difference test was used to compare multiple groups. $\mathrm{P}<0.05$ was considered to indicate a statistically significant difference. 
A

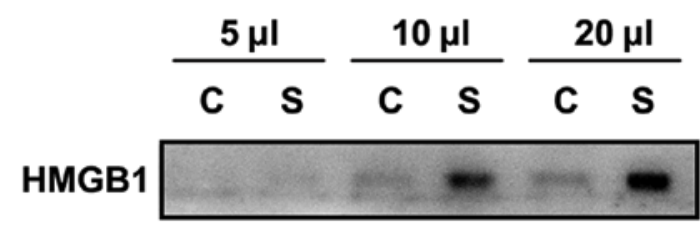

C

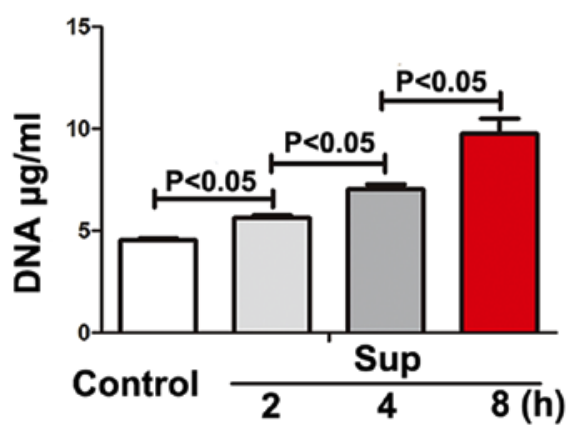

E
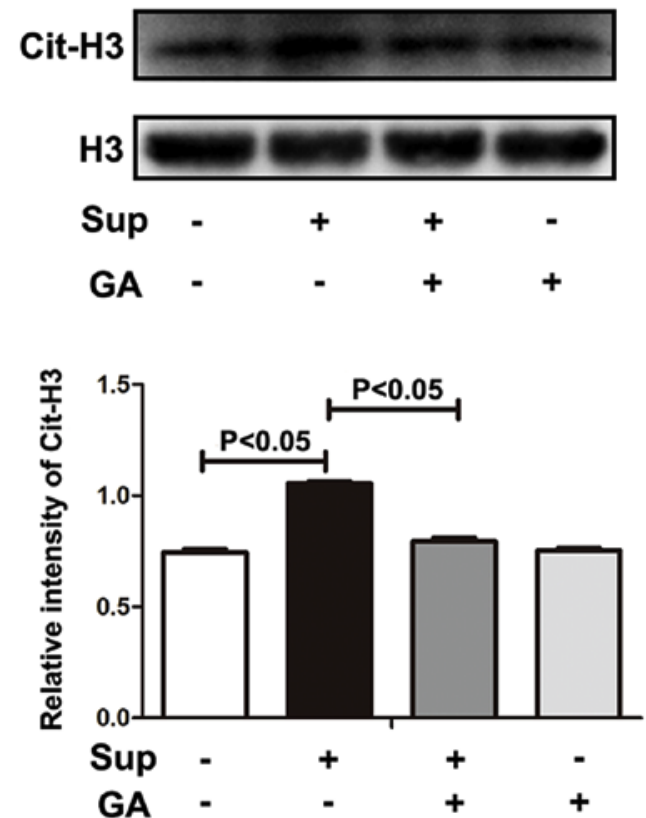

B

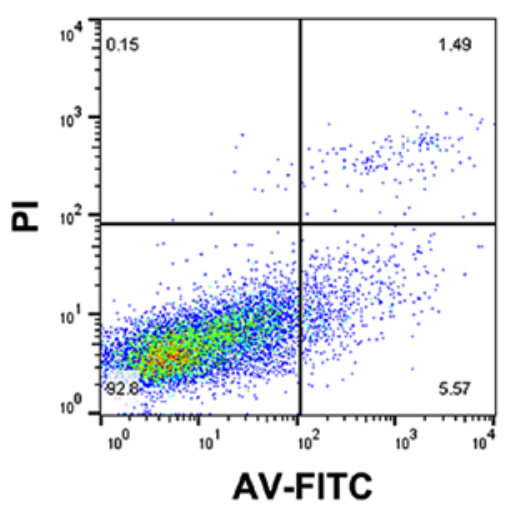

D

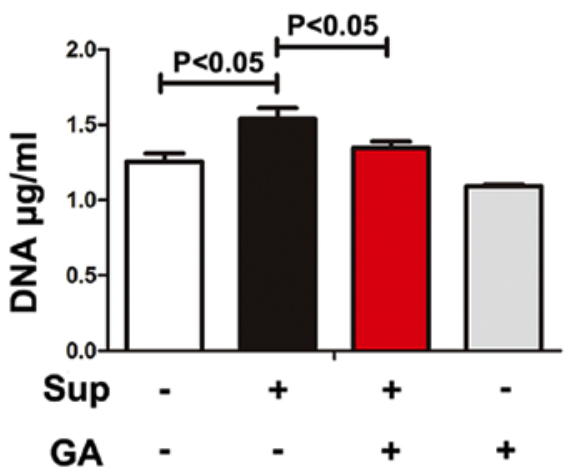

$\mathbf{F}$

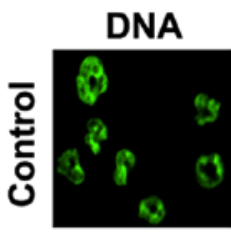

\section{Cit-H3}
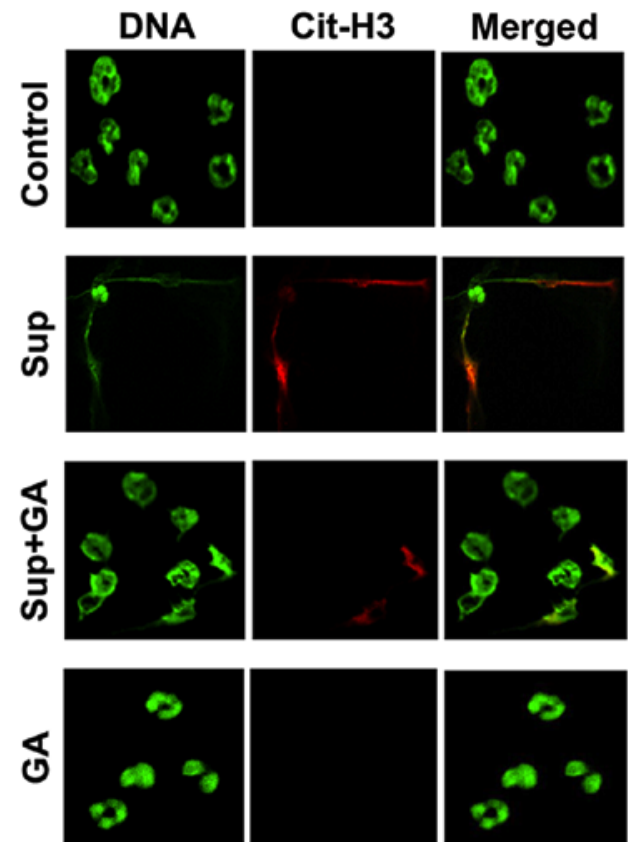

Figure 1. HMGB1 from LLC cells induces NETs. (A) HMGB1 from LLC supernatant was measured using western blot analysis. (B) Cell viability was evaluated using flow cytometry. (C) Neutrophils were treated with LLC supernatant and exDNA was detected using Sytox Green. (D) HMGB1 inhibitor decreased exDNA. (E) Levels of histone 3 and citrullinated histone 3 were measured using western blot analysis. Grayscale analysis verified the repeated results. (F) Images of NETs stained with Sytox Green and citrullinated histone 3 were captured using confocal microscopy. Magnification, 200x. LLC, Lewis lung cancer; HMGB1, high mobility group box 1; MLE, Murine Lung Epithelial; C, MLE cell culture supernatant; S/Sup, LLC culture supernatant; AV-FITC, Annexin-V-fluorescein isothiocyanate; PI, propidium iodide; exDNA, extracellular DNA; NETs, neutrophil extracellular traps; Cit-H3, citrullinated histone 3; GA, glycyrrhizic acid.

\section{Results}

HMGBI from LLC cells induces NETs. In the complete culture medium from LLC cells, HGMB1 was detected (Fig. 1A).
As expected, in the complete medium, only a small number of tumor cells underwent apoptosis or necrosis (Fig. 1B). Therefore, it was determined that LLC cells actively released HGMB1 without exogenous stimulus. 


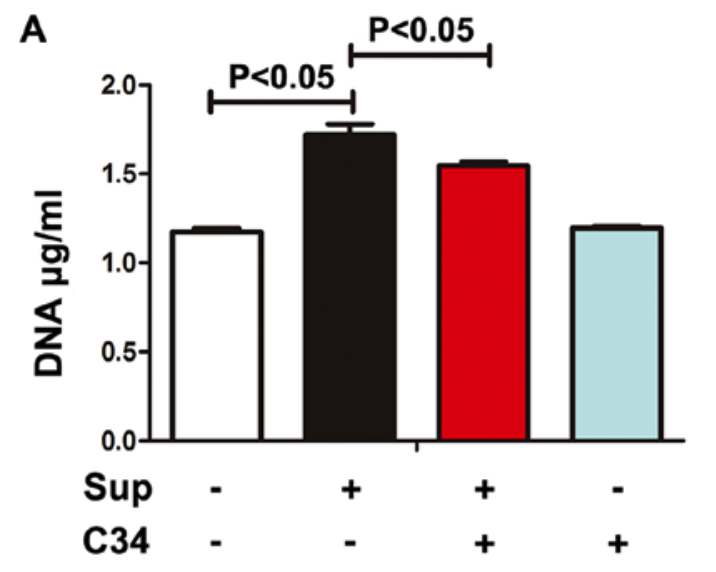

B
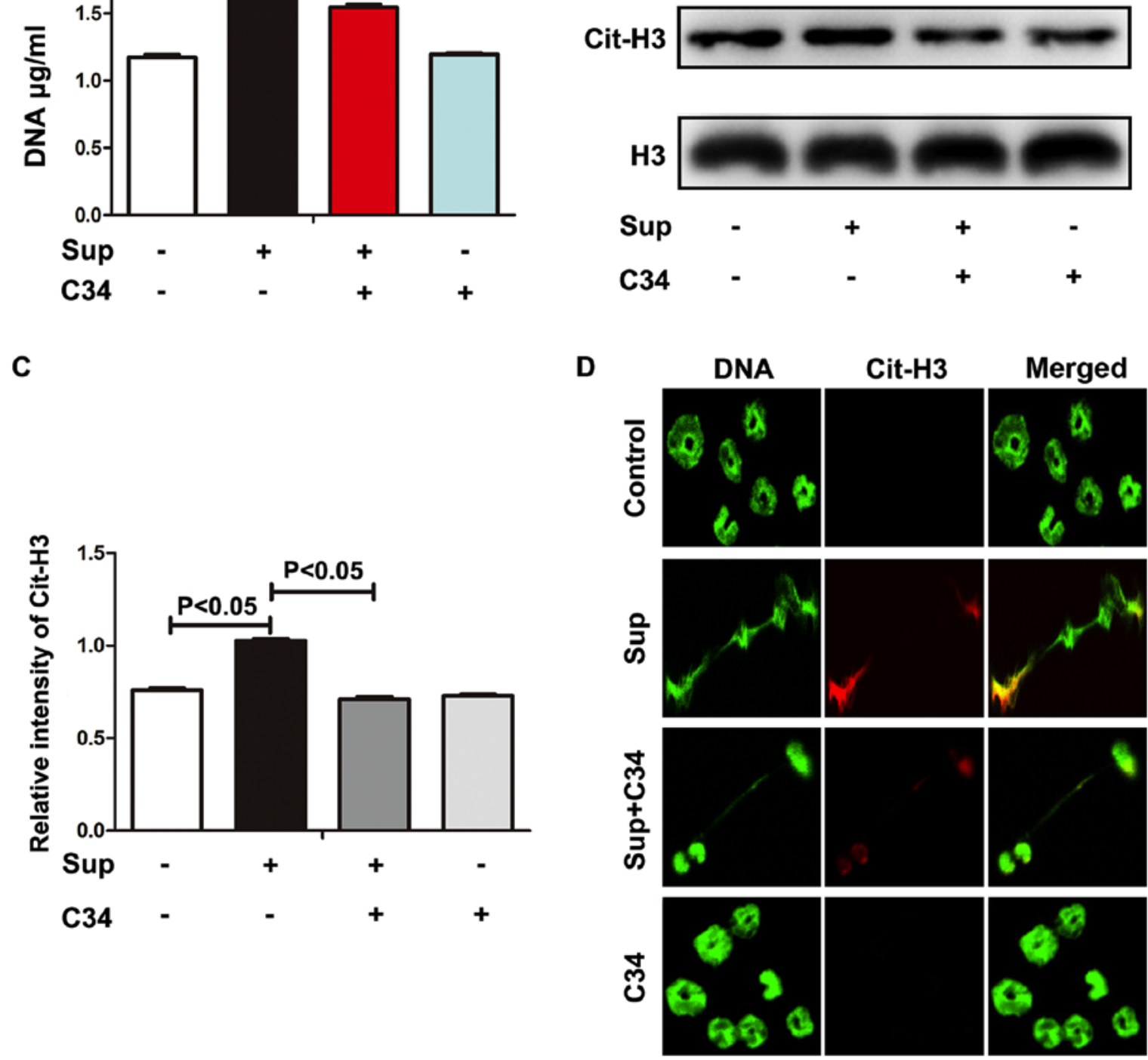

Figure 2. TLR4 is required for lung cancer cell-induced NETs. (A) TLR4 inhibitor C34 decreased extracellular DNA. (B) TLR4 inhibitor C34 decreased citrullinated histone 3. (C) Grayscale analysis verified these results. (D) NETs stained with Sytox Green and citrullinated histone 3 were captured under confocal microscopy. Magnification, 200x. HMGB1, high mobility group box 1; NETs, neutrophil extracellular traps; TLR4, Toll-like receptor 4; Cit-H3, citrullinated histone 3; Sup, supernatant of LLC cell culture.

As recombinant HMGB1 is able to induce NETs (12), it was hypothesized that LLC cell supernatants containing HMGB1 may also trigger the formation of NETs. Neutrophils were treated with LLC cell supernatants and the extracellular DNA (exDNA) was measured using DNA dye Sytox Green. As indicated in Fig. 1C, exDNA was progressively increased, suggesting that upon LLC cell supernatant challenge, neutrophils produced exDNA during the cell culture for $8 \mathrm{~h}$. To verify whether HMGB1 was involved with exDNA production, HMGB1 inhibitor GA was added to the neutrophil culture. GA significantly alleviated the exDNA production evoked by LLC cell supernatant (Fig. 1D), suggesting that the role of LLC cell supernatant in exDNA induction was at least partially dependent on HMGB1.

exDNA may originate from necrotic neutrophils or neutrophils with NETs. However, necrosis may be differentiated from neutrophils with NETs due to the observation of histone hypercitrullination in NETs (21). Therefore, histone hypercitrullination was evaluated in the neutrophils treated with LLC cell supernatant. As indicated in Fig. 1E, hypercitrullinated histone 3 expression was significantly increased in the neutrophils treated with LLC cell supernatant. In addition, HMGB1 inhibitor rescued the deleterious effects of LLC cell supernatant. Under confocal microscopy, LLC cell supernatant-treated neutrophils were observed to produce exDNA overlaid with hypercitrullinated histone 3 , which was alleviated by treatment with HMGB1 inhibitor GA (Fig. 1F). These results indicate that lung cancer cells actively release HMGB1, which directly promotes the formation of NETs.

TLR4 is required for lung cancer cell-induced NETs. As a damage-associated molecular pattern protein, soluble HMGB1 
A

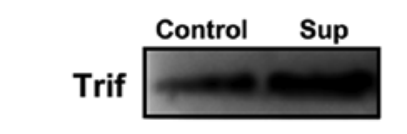

MyD88

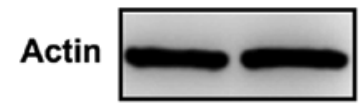

B

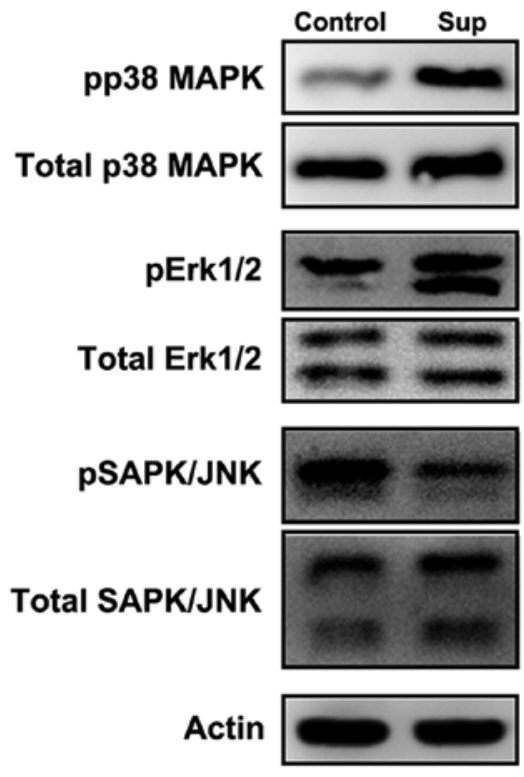

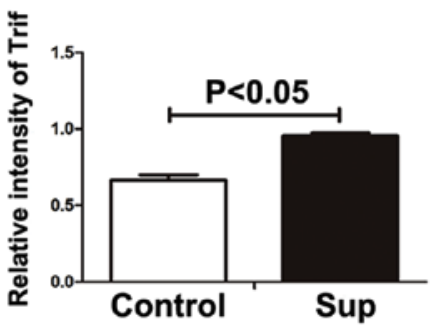

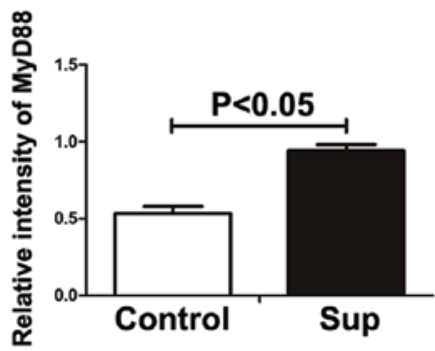

D

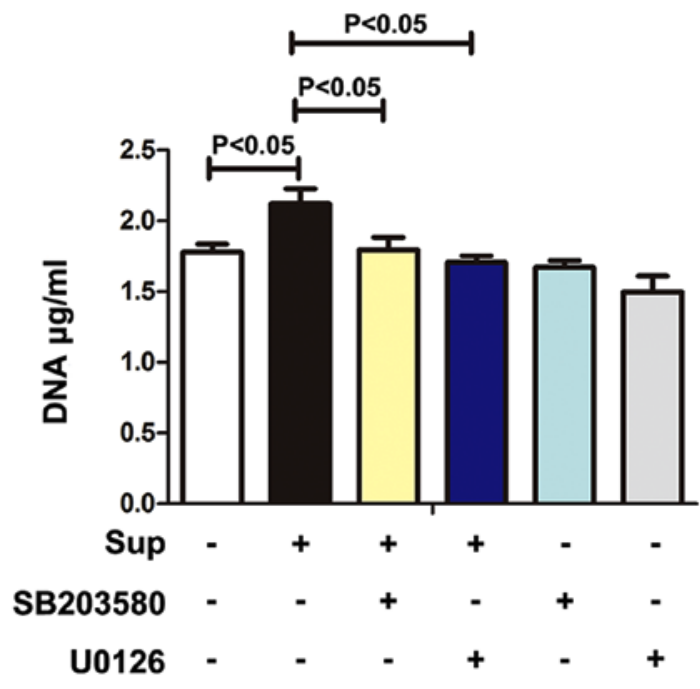

C
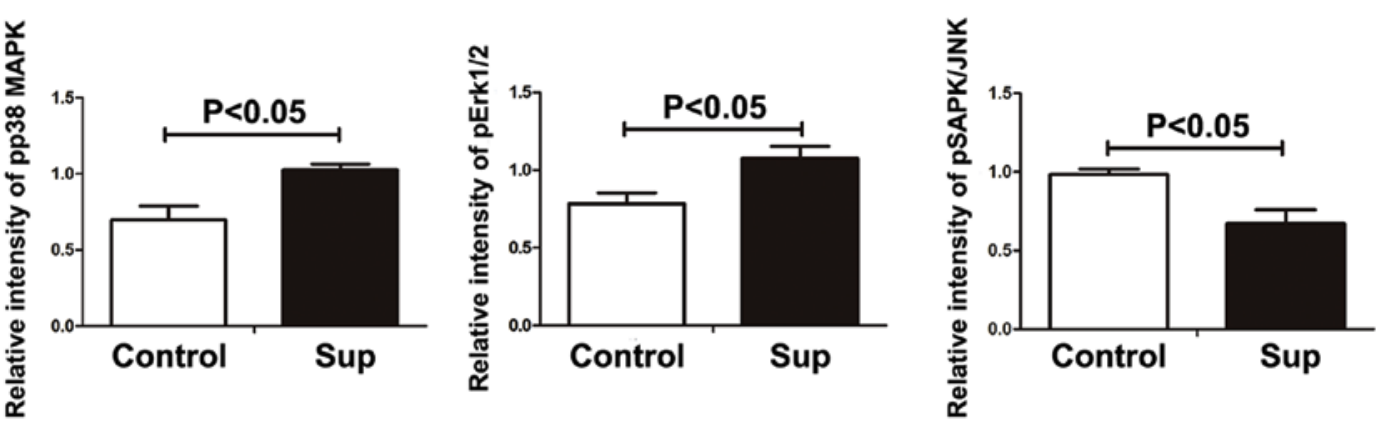

Figure 3. MAPK pathway is involved in lung cancer cell-induced NETs. (A) MyD88 and TRIF were measured using western blot analysis. Grayscale analysis confirmed repeated results. (B) MAPKs (P38, ERK and JNK) were measured using western blot analysis. (C) Grayscale analysis confirmed repeated results. (D) ERK inhibitor UO126 or p38 inhibitor SB203580 significantly decreased extracellular DNA. NETs, neutrophil extracellular traps; Trif, TIR-domain-containing adapter-inducing interferon- $\beta$; MyD88, myeloid differentiation factor 88; MAPK, mitogen-activated protein kinase; JNK, Janus kinase; p, phosphorylated; Sup, supernatant of LLC cell culture; SAPK, stress-activated protein kinase.

may bind with diverse receptors, including TLR4, the receptor for advanced glycation end products, macrophage adhesion molecule-1, receptor-type protein-tyrosine phosphatase- $\zeta / \beta$, chemokine (C-X-C motif) ligand 4, T-cell immunoglobulin mucin-3, cluster of differentiation 24 and syndecan 1 (11). Among these potential receptors, TLR4 is highly expressed on neutrophils (22) and closely associated with NETs. In bacterial sepsis, platelet TLR4 detected ligands and promoted NETs (7). Furthermore, NETs induced by recombinant HMGB1 were dependent on TLR4 (12). Therefore, in the present study, the role of TLR4 in LLC cell supernatant-induced NETs was explored. 
A

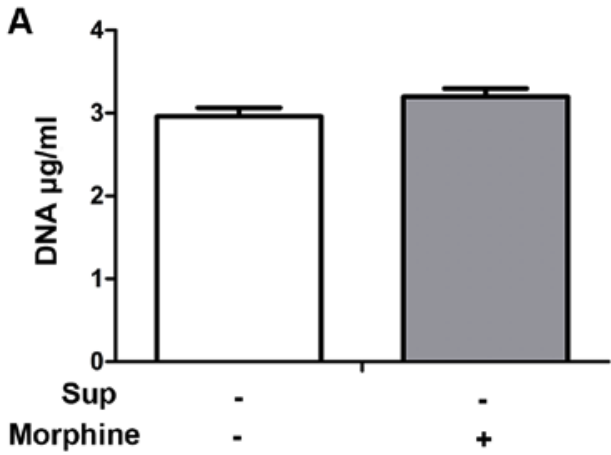

B
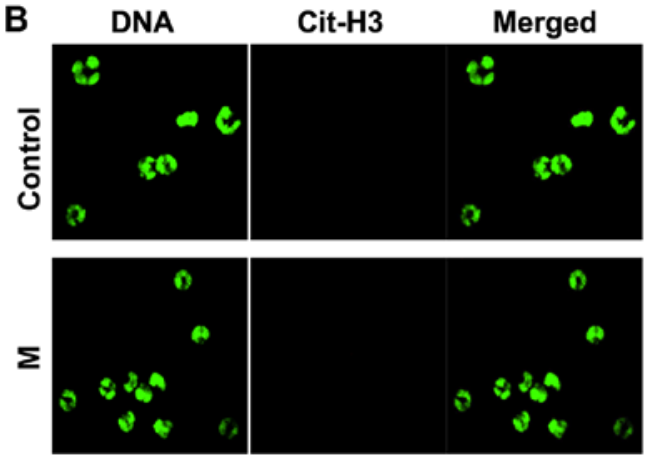

Figure 4. Morphine alone is not able to induce NETs. (A) Extracellular DNA from neutrophils was compared following treatment with or without morphine. (B) According to the immunofluorescence results, NETs were absent in the neutrophils stimulated with morphine alone. Magnification, 200x. NETs, neutrophil extracellular traps; Sup, supernatant of LLC cell culture; M, morphine; Cit-H3, citrullinated histone 3.

A

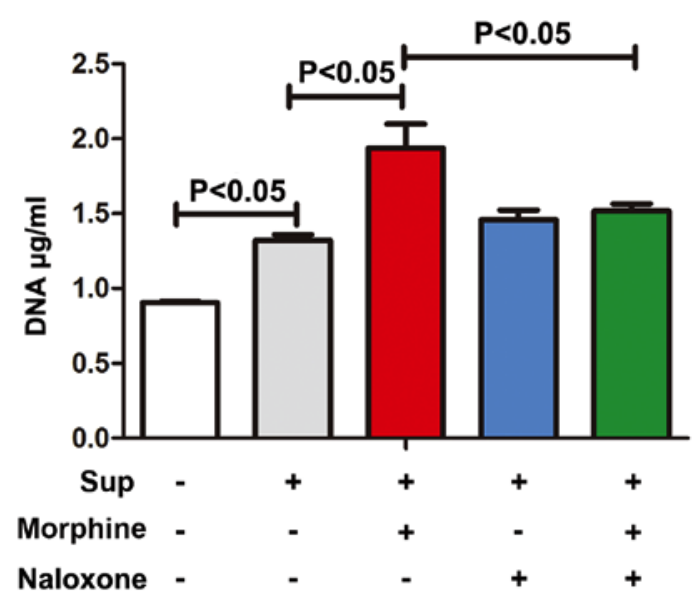

B
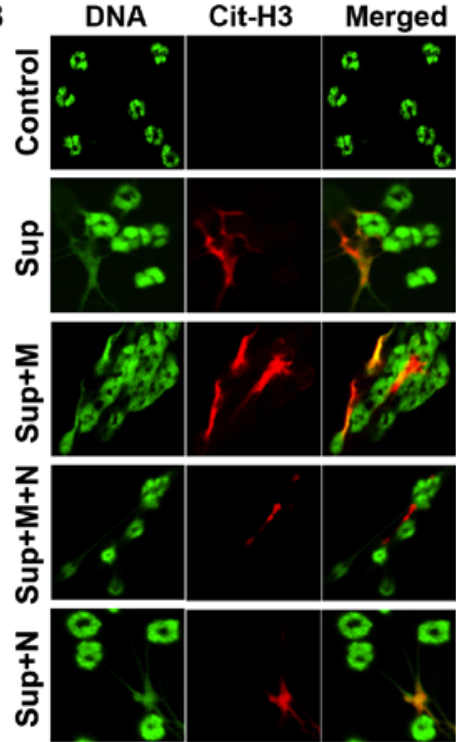

Figure 5. Morphine promotes lung cancer cell-induced NETs. (A) The levels of LLC cell supernatant-induced extracellular DNA were significantly increased following administration of morphine, and the effect was inhibited by naloxone. (B) NETs stained with Sytox Green and Cit-H3 were captured using confocal microscopy. Magnification, 200x. NETs, neutrophil extracellular traps; LLC, Lewis lung cancer; Sup, supernatant; Cit-H3, citrullinated Histone 3, M, morphine; N, naloxone.

C34 is a selective TLR4 inhibitor (23). ExDNA and histone 3 hypercitrullination were significantly decreased upon C34 treatment (Fig. 2A-C), suggesting that TLR4 may be required in LLC cell-induced NETs. Consistent with the observations of exDNA and histone 3 hypercitrullination, C34 also diminished NET formation as observed by confocal microscopy (Fig. 2D). Although C34 selectively targets TLR4 (23), TLR4 knockout neutrophils may be required to confirm whether LLC cell-induced NETs was via TLR4. Nevertheless, these results indicate that TLR4-HMBG1 may be required for lung cancer cell-induced NETs.

MAPK pathway is involved in lung cancer cell-induced NETs. Soluble HMGB1, once bound with TLR4, may trigger signal transduction via MyD88 or TRIF (24). As indicated in Fig. 3A, treatment with LLC cell supernatants resulted in the significant increase of MyD88 and TRIF. Once bound with the cytoplasmic portion of TLR4, Myd88 recruits nuclear factor- $\kappa$ B and MAPK (25), which have been demonstrated to be essential in NET formation $(26,27)$. As indicated in Fig. 3B and C, phosphorylation of p38 MAPKs, ERK or Janus kinase was significantly increased in the neutrophils treated with lung cancer cell supernatants. Furthermore, p38 MAPKs inhibitor sb203580 or ERK inhibitor U0126 significantly decreased the level of NETs induced by lung cancer cell supernatants (Fig. 3D), suggesting that p38 MAPKs and ERK were involved in lung cancer cell-induced NETs. These results indicate that HMGB1 induced NET formation via TLR4 and p38 MAPKs/ERK.

Morphine promotes lung cancer cell-induced NETs. The aforementioned results indicate that HMGB1 released from lung cancer cells induces NETs via the TLR4/MAPK signaling pathway. To alleviate cancer-associated pain, patients with lung cancer may be administered morphine, which also binds with TLR4 $(17,18)$. Therefore, neutrophils infiltrated into lung tissues may be stimulated by HMGB1 and morphine. To explore the combinational effects of morphine and HMGB1 
on NETs, neutrophils were treated with morphine and LLC cell supernatants. In the preliminary experiment, morphine alone did not evoke the formation of NETs (Fig. 4). However, morphine augmented the formation of NETs induced by lung cancer cell supernatants (Fig. 5). Naloxone, an antagonist of morphine, significantly inhibited the effect of morphine on NET induction, suggesting that opioid receptors may also be involved. In summary, these results indicate that morphine may promote lung cancer cell-induced NETs.

\section{Discussion}

Increased levels of HMGB1 are associated with increased disease severity in patients with non-small cell lung cancer $(28,29)$. In chemotherapy, HMGB1 passively released from necrotic cancer cells may increase invasion and metastasis. Cancer cells may also actively secrete HMGB1 upon exogenous and endogenous stimuli (11). Although HMGB1-stimulated NETs have been described previously (12), the role of this in cancer remains unclear. The present study provided evidence that HMGB1 from cancer cells may contribute to NET formation.

Once bound with neutrophil TLR4, HMGB1 induces the activation of Myd88 and TRIF. Although neutrophils express TRIF, it has been demonstrated that the TLR4 ligand lipopolysaccharide is not able to mobilize the TRIF signaling pathway, indicating that TRIF may not be directly involved with neutrophil TLR4 activation (30). In ischemia-reperfusion injury, HMGB1-TLR4-mediated acute cerebral infarct was identified to be TRIF-independent (31). Therefore, the present study focused on TLR4-Myd88 signal transduction initiated by HMGB1 in lung cancer cell supernatants. Activated platelets induced NETs in a pathway that involved TLR4 but was independent of p38 MAPKs (32). In inflammatory disease, oxidized low-density lipoprotein triggered the activation of $\mathrm{p} 38 \mathrm{MAPKs} / \mathrm{ERK}$ and the formation of NETs through TLR2 and TLR6 (33). TLR2/TLR6 is also able to bind with HMGB1 (34). Therefore, signal transduction in the formation of NETs may vary depending on the stimulus. In the present study, it was demonstrated that HMGB1 from lung cancer cells induced NETs, which was at least partially dependent on the TLR4 and p38 MAPKs/ERK signaling pathway.

As an analgesic for treating severe pain, morphine may suppress the immune response, impairing the function of $\mathrm{T}$ cells and macrophages (35). In addition, neutrophils from patients with sepsis are able to release endogenous morphine, which may inhibit inflammation (36). The present study aimed to explore whether morphine contributed to the formation of NETs. In combination with supernatants from lung cancer cells, morphine may aggravate the formation of NETs. In the end-stages of lung cancer, HMGB1 from lung cancer cells and exogenous morphine administration may synergistically fuel the formation of NETs and cancer progression. It would be useful to investigate whether lung cancer cells are able to release endogenous morphine. Future studies will investigate the association between morphine and NETs in greater detail.

In the infiltrated inflammatory cells within the tumor microenvironment, tumor-associated neutrophils confer a poor prognosis (37). In breast cancer, G-CSF-induced NETs facilitate metastasis (38). As HMGB1 from lung cancer cells and morphine have been indicated to promote the formation of NETs, it is postulated that targeting NETs and their initiators, including HMGB1 and morphine, may be valuable in cancer therapy.

The present study contains certain limitations. Firstly, the mechanisms through which lung cancer cells actively release HMGB1 were not explored. Secondly, the effects of NETs in vivo were not assessed. Thirdly, NETs formation in the patients with lung cancer with or without morphine treatment was not compared. However, the observations from the present study clearly indicated that HMGB1 from lung cancer cells and morphine contributed to the NETs formation, which may provide additional information concerning the tumorigenesis of lung cancer.

\section{Acknowledgements}

Not applicable.

\section{Funding}

The present study was supported by National Natural Science Foundation of China (grant no. 81671563), Natural Science Foundation of Jiangsu Province (grant no. BK2015155) and Nanjing Medical University key project (grant no. 2014NJMUZD010).

\section{Availability of data and materials}

The datasets used and/or analyzed during the present study are available from the corresponding author on reasonable request.

\section{Authors' contributions}

YC and MZ conceived and designed the study. JZ, YY, TG and YL conducted the experiments; FH, NH, BY and MZ analyzed the results. All authors reviewed and approved the manuscript.

\section{Ethics approval and consent to participate}

All animal procedures were approved by the Institutional Animal Care Committee of Nanjing Medical University.

\section{Patient consent for publication}

Not applicable.

\section{Competing interests}

The authors declare that they have no competing interests.

\section{References}

1. Bar-Ad V, Palmer J, Li L, Lai Y, Lu B, Myers RE, Ye Z, Axelrod R, Johnson JM, Werner-Wasik M, et al: Neutrophil to lymphocyte ratio associated with prognosis of lung cancer. Clin Transl Oncol 19: 711-717, 2017.

2. Najmeh S, Cools-Lartigue J, Giannias B, Spicer J and Ferri LE Simplified human neutrophil extracellular traps (NETs) isolation and handling. J Vis Exp, 2015. 
3. Cools-Lartigue J, Spicer J, McDonald B, Gowing S, Chow S, Giannias B, Bourdeau F, Kubes P and Ferri L: Neutrophil extracellular traps sequester circulating tumor cells and promote metastasis. J Clin Invest: July 1, 2013 (Epub ahead of print).

4. Oklu R, Sheth RA, Wong KHK, Jahromi AH and Albadawi H: Neutrophil extracellular traps are increased in cancer patients but does not associate with venous thrombosis. Cardiovasc Diagn Ther 7 (Suppl 3): S140-S149, 2017.

5. Brinkmann V, Reichard U, Goosmann C, Fauler B, Uhlemann Y, Weiss DS, Weinrauch Y and Zychlinsky A: Neutrophil extracellular traps kill bacteria. Science 303: 1532-1535, 2004.

6. Ben-Smith A, Dove SK, Martin A, Wakelam MJ and Savage CO Antineutrophil cytoplasm autoantibodies from patients with systemic vasculitis activate neutrophils through distinct signaling cascades: Comparison with conventional Fcgamma receptor ligation. Blood 98: 1448-1455, 2001.

7. Clark SR, Ma AC, Tavener SA, McDonald B, Goodarzi Z, Kelly MM,Patel KD, Chakrabarti S, McAvoy E, Sinclair GD, et al: Platelet TLR4 activates neutrophil extracellular traps to ensnare bacteria in septic blood. Nat Med 13: 463-469, 2007.

8. Mitroulis I, Kambas K, Chrysanthopoulou A, Skendros P, Apostolidou E, Kourtzelis I, Drosos GI, Boumpas DT and Ritis K: Neutrophil extracellular trap formation is associated with IL-1beta and autophagy-related signaling in gout. PLoS One 6: e29318, 2011.

9. Gupta AK, Joshi MB, Philippova M, Erne P, Hasler P, Hahn S and Resink TJ: Activated endothelial cells induce neutrophi extracellular traps and are susceptible to NETosis-mediated cell death. FEBS Lett 584: 3193-3197, 2010.

10. Demers M, Krause DS, Schatzberg D, Martinod K, Voorhees JR, Fuchs TA, Scadden DT and Wagner DD: Cancers predispose neutrophils to release extracellular DNA traps that contribute to cancer-associated thrombosis. Proc Natl Acad Sci USA 109: 13076-13081, 2012.

11. Kang R, Zhang Q, Zeh HJ III, Lotze MT and Tang D: HMGB1 in cancer: Good, bad, or both? Clin Cancer Res 19: 4046-4057, 2013.

12. Tadie JM, Bae HB, Jiang S, Park DW, Bell CP, Yang H, Pittet JF Tracey K, Thannickal VJ, Abraham E and Zmijewski JW: HMGB1 promotes neutrophil extracellular trap formation through interactions with Toll-like receptor 4. Am J Physiol Lung Cell Mol Physiol 304: L342-L349, 2013.

13. Kim YH, Okuda C, Sakamori Y, Masago K, Togashi Y and Mishima M: Continuous morphine infusion for end-stage lung cancer patients. Oncol Lett 5: 972-974, 2013

14. Zylla D, Kuskowski MA, Gupta K and Gupta P: Association of opioid requirement and cancer pain with survival in advanced non-small cell lung cancer. Br J Anaesth 113 (Suppl 1): i109-i116, 2014.

15. Nguyen J, Luk K, Vang D, Soto W, Vincent L, Robiner S, Saavedra R, Li Y, Gupta P and Gupta K: Morphine stimulates cancer progression and mast cell activation and impairs survival in transgenic mice with breast cancer. Br J Anaesth 113 (Suppl 1) i4-13, 2014

16. Zylla D, Gourley BL, Vang D, Jackson S, Boatman S, Lindgren B, Kuskowski MA, Le C, Gupta K and Gupta P: Opioid requirement, opioid receptor expression, and clinical outcomes in patients with advanced prostate cancer. Cancer 119: 4103-4110, 2013.

17. Shah M, Anwar MA, Yesudhas D, Krishnan J and Choi S: A structural insight into the negative effects of opioids in analgesia by modulating the TLR4 signaling: An in silico approach. Sci Rep 6: 39271, 2016.

18. Shah M and Choi S: Toll-like Receptor-Dependent Negative Effects of Opioids: A Battle between Analgesia and Hyperalgesia. Front Immunol 8: 642, 2017.

19. Swamydas M, Luo Y, Dorf ME and Lionakis MS: Isolation of mouse neutrophils. Curr Protoc Immunol 110: 3.20.1-3.15, 2015.

20. Behnen M, Leschczyk C, Moller S, Batel T, Klinger M, Solbach $\mathrm{W}$ and Laskay T: Immobilized immune complexes induce neutrophil extracellular trap release by human neutrophil granulocytes via Fc gamma RIIIB and Mac-1. J Immunol 193 1954-1965, 2014.
21. Brinkmann V and Zychlinsky A: Neutrophil extracellular traps: Is immunity the second function of chromatin? J Cell Biol 198: 773-783, 2012.

22. Hayashi F, Means TK and Luster AD: Toll-like receptors stimulate human neutrophil function. Blood 102: 2660-2669, 2003.

23. Neal MD, Jia H, Eyer B, Good M, Guerriero CJ, Sodhi CP, Afrazi A, Prindle T Jr, Ma C, Branca M, et al: Discovery and validation of a new class of small molecule Toll-like receptor 4 (TLR4) inhibitors. PLoS One 8: e65779, 2013.

24. Reino DC, Pisarenko V, Palange D, Doucet D, Bonitz RP, Lu Q, Colorado I, Sheth SU, Chandler B, Kannan KB, et al: Trauma hemorrhagic shock-induced lung injury involves a gut-lymph-induced TLR4 pathway in mice. PLoS One 6: e14829, 2011.

25. Takeda K and Akira S: TLR signaling pathways. Semin Immunol 16: 3-9, 2004.

26. Lapponi MJ, Carestia A, Landoni VI, Rivadeneyra L, Etulain J, Negrotto S, Pozner RG and Schattner M: Regulation of neutrophil extracellular trap formation by anti-inflammatory drugs. J Pharmacol Exp Ther 345: 430-437, 2013.

27. Hakkim A,Fuchs TA, Martinez NE,Hess S, Prinz H,Zychlinsky A and Waldmann H: Activation of the Raf-MEK-ERK pathway is required for neutrophil extracellular trap formation. Nat Chem Biol 7: 75-77, 2011

28. Ma Y, Kang S, Wu X, Han B, Jin Z and Guo Z: Up-regulated HMGB1 in the pleural effusion of non-small cell lung cancer (NSCLC) patients reduces the chemosensitivity of NSCLC cells. Tumori 104: 338-343, 2018.

29. Niki M, Yokoi T, Kurata T and Nomura S: New prognostic biomarkers and therapeutic effect of bevacizumab for patients with non-small-cell lung cancer. Lung Cancer (Auckl) 8: 91-99, 2017.

30. Tamassia N, Le Moigne V, Calzetti F, Donini M, Gasperini S, Ear T, Cloutier A, Martinez FO, Fabbri M, Locati M, et al: The MyD88-independent pathway is not mobilized in human neutrophils stimulated via TLR4. J Immunol 178: 7344-7356, 2007.

31. Yang QW, Lu FL, Zhou Y, Wang L, Zhong Q, Lin S, Xiang J, Li JC, Fang CQ and Wang JZ: HMBG1 mediates ischemia-reperfusion injury by TRIF-adaptor independent Toll-like receptor 4 signaling. J Cereb Blood Flow Metab 31: 593-605, 2011.

32. Carestia A, Kaufman T, Rivadeneyra L, Landoni VI, Pozner RG, Negrotto S, D'Atri LP, Gómez RM and Schattner M: Mediators and molecular pathways involved in the regulation of neutrophil extracellular trap formation mediated by activated platelets. J Leukoc Biol 99: 153-162, 2016.

33. Awasthi D, Nagarkoti S, Kumar A, Dubey M, Singh AK, Pathak P, Chandra T, Barthwal MK and Dikshit M: Oxidized LDL induced extracellular trap formation in human neutrophils via TLR-PKC-IRAK-MAPK and NADPH-oxidase activation. Free Radic Biol Med 93: 190-203, 2016.

34. Yu M, Wang H, Ding A, Golenbock DT, Latz E, Czura CJ, Fenton MJ, Tracey KJ and Yang H: HMGB1 signals through toll-like receptor (TLR) 4 and TLR2. Shock 26: 174-179, 2006.

35. Plein LM and Rittner HL: Opioids and the immune system-friend or foe. Br J Pharmacol 175: 2717-2725, 2017.

36. Glattard E, Welters ID, Lavaux T, Muller AH, Laux A, Zhang D, Schmidt AR, Delalande F, Laventie BJ, Dirrig-Grosch S, et al: Endogenous morphine levels are increased in sepsis: A partial implication of neutrophils. PLoS One 5: e8791, 2010.

37. Gregory AD and Houghton AM: Tumor-associated neutrophils: new targets for cancer therapy. Cancer Res 71: 2411-2416, 2011.

38. Berger-Achituv S, Brinkmann V, Abed UA, Kuhn LI, Ben-Ezra J, Elhasid R and Zychlinsky A: A proposed role for neutrophil extracellular traps in cancer immunoediting. Front Immunol 4: $48,2013$.

c) (7) $\odot$ This work is licensed under a Creative Commons Attribution-NonCommercial-NoDerivatives 4.0 International (CC BY-NC-ND 4.0) License. 